УДК 517.98

\author{
T. V. Vasylyshyn, A. V. Zagorodnyuk
}

\title{
SYMMETRIC POLYNOMIALS ON THE CARTESIAN POWER OF THE REAL BANACH SPACE $L_{\infty}[0,1]$
}

\begin{abstract}
T. V. Vasylyshyn, A. V. Zagorodnyuk. Symmetric polynomials on the Cartesian power of the real Banach space $L_{\infty}[0,1]$, Mat. Stud. 53 (2020), 192-205.

We construct an algebraic basis of the algebra of symmetric (invariant under composition of the variable with any measure preserving bijection of $[0,1]$ ) continuous polynomials on the $n$th Cartesian power of the real Banach space $L_{\infty}^{(\mathbb{R})}[0,1]$ of Lebesgue measurable essentially bounded real valued functions on $[0,1]$. Also we describe the spectrum of the Fréchet algebra $\mathcal{A}_{s}\left(L_{\infty}^{(\mathbb{R})}[0,1]\right)$ of symmetric real-valued functions on the space $L_{\infty}^{(\mathbb{R})}[0,1]$, which is the completion of the algebra of symmetric continuous real-valued polynomials on $L_{\infty}^{(\mathbb{R})}[0,1]$ with respect to the family of norms of uniform convergence of complexifications of polynomials. We show that $\mathcal{A}_{s}\left(L_{\infty}^{(\mathbb{R})}[0,1]\right)$ contains not only analytic functions. Results of the paper can be used for investigations of algebras of symmetric functions on the $n$th Cartesian power of the Banach space $L_{\infty}^{(\mathbb{R})}[0,1]$.
\end{abstract}

Introduction. Symmetric polynomials on infinite-dimensional Banach spaces were studied, firstly, by Nemirovski and Semenov in [9]. In particular, in [9] there were constructed algebraic bases (see definition below) of the algebra of symmetric (invariant under composition of the variable with any measure preserving bijection of $[0,1]$ ) continuous real-valued polynomials on the real Banach space $L_{p}[0,1]$, where $1 \leq p<+\infty$, of Lebesgue measurable integrable in a power $p$ real-valued functions on $[0,1]$ and the algebra of symmetric (invariant under any permutation of elements of the variable) continuous real-valued polynomials on the real Banach space $\ell_{p}$, where $1 \leq p<+\infty$, of sequences of real numbers such that the series of absolute values of its elements in a power $p$ is convergent. These results were generalized to separable rearrangement-invariant real Banach spaces in [5]. Some results for symmetric polynomials and symmetric analytic functions on nonseparable complex Banach spaces were established in [2, 3, 4]. In particular, in [2] it was constructed an algebraic basis of the algebra of symmetric continuous complex-valued polynomials on the complex Banach space $L_{\infty}^{(\mathbb{C})}[0,1]$ of Lebesgue measurable essentially bounded complex-valued functions on $[0,1]$ and it was described the spectrum of the Fréchet algebra $H_{b s}\left(L_{\infty}^{(\mathbb{C})}[0,1]\right)$ of symmetric analytic complexvalued functions on $L_{\infty}^{(\mathbb{C})}[0,1]$, which are bounded on bounded sets of $L_{\infty}^{(\mathbb{C})}[0,1]$. In [4] it was shown that the algebra $H_{b s}\left(L_{\infty}^{(\mathbb{C})}[0,1]\right)$ is isomorphic to the algebra of all analytic functions on the strong dual of the space of entire functions on the complex plane $\mathbb{C}$. In [3] it was shown that there exist only trivial complex-valued symmetric continuous polynomials on the

2020 Mathematics Subject Classification: 46G25, 46E15, 46E25, 46G20.

Keywords: symmetric polynomial; spectrum of the Fréchet algebra.

doi:10.30970/ms.53.2.192-205

(C) T. V. Vasylyshyn, A. V. Zagorodnyuk, 2020 
complex Banach space of Lebesgue measurable essentially bounded complex-valued functions on the semi-axis. Symmetric polynomials and symmetric analytic functions on Cartesian products of some complex Banach spaces were studied in $[6,7,10,11,12]$. In particular, in [12] it was constructed an algebraic basis of the algebra of symmetric continuous complexvalued polynomials on the $n$th Cartesian power of the space $L_{\infty}^{(\mathbb{C})}[0,1]$. Note that the results of [12] cannot be literally rewritten to the real case, because most of these results essentially use [2, Theorem 3.1] and [7, Theorem 6], which are not valid in the real case.

In this work, we construct an algebraic basis of the algebra of symmetric continuous realvalued polynomials on the $n$th Cartesian power of the real Banach space $L_{\infty}^{(\mathbb{R})}[0,1]$ of Lebesgue measurable essentially bounded real-valued functions on $[0,1]$. Also we describe the spectrum of the Fréchet algebra $\mathcal{A}_{s}\left(L_{\infty}^{(\mathbb{R})}[0,1]\right)$ of symmetric real-valued functions on the space $L_{\infty}^{(\mathbb{R})}[0,1]$, which is the completion of the algebra of symmetric continuous real-valued polynomials on $L_{\infty}^{(\mathbb{R})}[0,1]$ with respect to the family of norms of uniform convergence of complexifications of polynomials. We show that the restriction of any symmetric analytic function of bounded type on the complex Banach space $L_{\infty}^{(\mathbb{C})}[0,1]$ of Lebesgue measurable essentially bounded complex-valued functions on $[0,1]$ with real coefficients to $L_{\infty}^{(\mathbb{R})}[0,1]$ belongs to $\mathcal{A}_{s}\left(L_{\infty}^{(\mathbb{R})}[0,1]\right)$. On the other hand, the function

$$
L_{\infty}^{(\mathbb{R})}[0,1] \ni y \mapsto\left|\int_{[0,1]} y(t) d t\right| \in \mathbb{R}
$$

belongs to $\mathcal{A}_{s}\left(L_{\infty}^{(\mathbb{R})}[0,1]\right)$, but it is not analytic.

1. Preliminaries. We denote by $\mathbb{N}$ the set of all positive integers and by $\mathbb{Z}_{+}$the set of all nonnegative integers.

A mapping $P: X \rightarrow Y$, where $X$ and $Y$ are Banach spaces with norms $\|\cdot\|_{X}$ and $\|\cdot\|_{Y}$ respectively, is called an $N$-homogeneous polynomial, where $N \in \mathbb{N}$, if there exists an $N$-linear symmetric mapping $A_{P}: X^{N} \rightarrow Y$ such that

$$
P(x)=A_{P}(\underbrace{x, \ldots, x}_{N})
$$

for every $x \in X$. Here "symmetric" means that $A_{P}\left(x_{\tau(1)}, \ldots, x_{\tau(N)}\right)=A_{P}\left(x_{1}, \ldots, x_{N}\right)$ for every permutation $\tau:\{1, \ldots, N\} \rightarrow\{1, \ldots, N\}$. The mapping $A_{P}$ is called the $N$-linear symmetric mapping associated with $P$.

It is known (see e.g. [8], Theorem 1.10) that $A_{P}$ can be recovered from $P$ by means of the so-called Polarization Formula:

$$
A_{P}\left(x_{1}, \ldots, x_{N}\right)=\frac{1}{N ! 2^{N}} \sum_{\varepsilon_{1}, \ldots, \varepsilon_{N}= \pm 1} \varepsilon_{1} \ldots \varepsilon_{N} P\left(\varepsilon_{1} x_{1}+\ldots+\varepsilon_{N} x_{N}\right) .
$$

It is known that an $N$-homogeneous polynomial $P: X \rightarrow Y$ is continuous if and only if

$$
\|P\|=\sup _{\|x\|_{X} \leq 1}\|P(x)\|_{Y}<+\infty .
$$

Similarly, an $N$-linear mapping $A: X^{N} \rightarrow Y$ is continuous if and only if

$$
\|A\|=\sup _{\left\|x_{1}\right\|_{X} \leq 1, \ldots,\left\|x_{N}\right\|_{X} \leq 1}\left\|A\left(x_{1}, \ldots, x_{N}\right)\right\|_{Y}<+\infty .
$$


Formula (1) implies the following inequality, which is called the Polarization Inequality:

$$
\|P\| \leq\left\|A_{P}\right\| \leq \frac{N^{N}}{N !}\|P\|
$$

for every continuous $N$-homogeneous polynomial $P$.

A mapping $P: X \rightarrow Y$ is called a polynomial of degree at most $N$ if it can be represented in the form

$$
P=P_{0}+P_{1}+\ldots+P_{N},
$$

where $P_{0} \in Y$ and $P_{j}: X \rightarrow Y$ is a $j$-homogeneous polynomial for every $j \in\{1, \ldots, N\}$. Let $\lambda_{0}, \ldots, \lambda_{N}$ be arbitrary distinct numbers. Note that

$$
P\left(\lambda_{j} x\right)=P_{0}+\lambda_{j} P_{1}(x)+\ldots+\lambda_{j}^{N} P_{N}(x)
$$

for every $j \in\{0, \ldots, N\}$ and $x \in X$. Therefore,

$$
\left(P\left(\lambda_{0} x\right), \ldots, P\left(\lambda_{N} x\right)\right)^{T}=V_{\lambda_{0}, \ldots, \lambda_{N}}\left(P_{0}, P_{1}(x), \ldots, P_{N}(x)\right)^{T},
$$

where $V_{\lambda_{0}, \ldots, \lambda_{N}}$ is a Vandermonde matrix, defined by $V_{\lambda_{0}, \ldots, \lambda_{N}}=\left(\lambda_{j}^{k}\right)_{j, k=\overline{0, N}}$. Since $\operatorname{det} V_{\lambda_{0}, \ldots, \lambda_{N}}$, is not equal to zero, it follows that there exists the inverse matrix $V_{\lambda_{0}, \ldots, \lambda_{N}}^{-1}=\left(w_{j k}\right)_{j, k=\overline{0, N}}$. Consequently,

$$
\left(P_{0}, P_{1}(x), \ldots, P_{N}(x)\right)^{T}=V_{\lambda_{0}, \ldots, \lambda_{N}}^{-1}\left(P\left(\lambda_{0} x\right), \ldots, P\left(\lambda_{N} x\right)\right)^{T}
$$

i.e.,

$$
P_{j}(x)=\sum_{k=0}^{N} w_{j k} P\left(\lambda_{k} x\right),
$$

for every $j \in\{0, \ldots, N\}$ and $x \in X$. Note that the equality (3) is called the Martin formula.

Let $\mathbb{K}=\mathbb{R}$ or $\mathbb{C}$. Let $L_{\infty}^{(\mathbb{K})}[0,1]$ be the Banach space over the field $\mathbb{K}$ of all Lebesgue measurable essentially bounded $\mathbb{K}$-valued functions $y$ on $[0,1]$ with norm

$$
\|y\|_{\infty}=\operatorname{ess~sup}_{t \in[0,1]}|y(t)|
$$

Let $n \in \mathbb{N}$. Let $\left(L_{\infty}^{(\mathbb{K})}[0,1]\right)^{n}$ be the $n$th Cartesian power of $L_{\infty}^{(\mathbb{K})}[0,1]$ with norm

$$
\|y\|_{\infty, n}=\max _{1 \leq s \leq n}\left\|y_{s}\right\|_{\infty}
$$

where $y=\left(y_{1}, \ldots, y_{n}\right) \in\left(L_{\infty}^{(\mathbb{K})}[0,1]\right)^{n}$.

Let $\Xi$ be the set of all bijections $\sigma:[0,1] \rightarrow[0,1]$ such that both $\sigma$ and $\sigma^{-1}$ are measurable and preserve the Lebesgue measure. A function $f:\left(L_{\infty}^{(\mathbb{K})}[0,1]\right)^{n} \rightarrow \mathbb{K}$ is called symmetric if

$$
f(y \circ \sigma)=f(y)
$$

for every $y=\left(y_{1}, \ldots, y_{n}\right) \in\left(L_{\infty}^{(\mathbb{K})}[0,1]\right)^{n}$ and for every $\sigma \in \Xi$, where $y \circ \sigma=\left(y_{1} \circ \sigma, \ldots, y_{n} \circ \sigma\right)$.

Formula (1) implies the following corollary. 
Corollary 1. Let $P:\left(L_{\infty}^{(\mathbb{K})}[0,1]\right)^{n} \rightarrow \mathbb{K}$ be a symmetric $N$-homogeneous polynomial. Then

$$
A_{P}\left(y_{1} \circ \sigma, \ldots, y_{N} \circ \sigma\right)=A_{P}\left(y_{1}, \ldots, y_{N}\right)
$$

for every $y_{1}, \ldots, y_{N} \in\left(L_{\infty}^{(\mathbb{K})}[0,1]\right)^{n}$ and $\sigma \in \Xi$.

For every multi-index $k=\left(k_{1}, \ldots, k_{n}\right) \in \mathbb{Z}_{+}^{n}$ such that $|k| \geq 1$, where $|k|=k_{1}+\ldots+k_{n}$, let us define a mapping $R_{k}^{(\mathbb{K})}:\left(L_{\infty}^{(\mathbb{K})}[0,1]\right)^{n} \rightarrow \mathbb{K}$ by

$$
R_{k}^{(\mathbb{K})}(y)=\int_{[0,1]} \prod_{\substack{s=1 \\ k_{s}>0}}^{n}\left(y_{s}(t)\right)^{k_{s}} d t
$$

where $y=\left(y_{1}, \ldots, y_{n}\right) \in\left(L_{\infty}^{(\mathbb{K})}[0,1]\right)^{n}$. Note that $R_{k}^{(\mathbb{K})}$ is a continuous symmetric $|k|$-homogeneous polynomial and $\left\|R_{k}^{(\mathbb{K})}\right\|=1$.

Let $\Omega$ be an arbitrary nonempty set. A mapping $f: \Omega \rightarrow \mathbb{K}$ is called an algebraic combination of mappings $f_{1}, \ldots, f_{m}: \Omega \rightarrow \mathbb{K}$ if there exists a polynomial $Q: \mathbb{K}^{m} \rightarrow \mathbb{K}$ such that

$$
f(x)=Q\left(f_{1}(x), \ldots, f_{m}(x)\right)
$$

for every $x \in \Omega$. A set $\left\{f_{1}, \ldots, f_{m}\right\}$ of mappings $f_{1}, \ldots, f_{m}: \Omega \rightarrow \mathbb{K}$ is called algebraically independent if

$$
Q\left(f_{1}(x), \ldots, f_{m}(x)\right)=0
$$

for every $x \in \Omega$ if and only if the polynomial $Q$ is identically equal to zero. If a set of mappings $\left\{f_{1}, \ldots, f_{k}\right\}$ is algebraically independent and polynomials $Q_{1}, Q_{2}: \mathbb{K}^{m} \rightarrow \mathbb{K}$ are such that

$$
Q_{1}\left(f_{1}(x), \ldots, f_{m}(x)\right)=Q_{2}\left(f_{1}(x), \ldots, f_{m}(x)\right)
$$

for every $x \in \Omega$, then the polynomial $Q_{1}$ is identically equal to the polynomial $Q_{2}$. Thus, every algebraic combination of elements of an algebraically independent set of mappings is unique. An infinite set of mappings is called algebraically independent if every its finite subset is algebraically independent. A subset $\mathcal{B}$ of some algebra of mappings $\mathcal{A}$ is called an algebraic basis of $\mathcal{A}$ if every element of $\mathcal{A}$ can be uniquely represented as an algebraic combination of some elements of $\mathcal{B}$. Evidently, every algebraic basis is algebraically independent.

For every nonempty finite set $M \subset \mathbb{Z}_{+}^{n}$ and for every mapping $l: M \rightarrow \mathbb{Z}_{+}$, let

$$
\varkappa(l, M)=\sum_{k \in M}|k| l(k) .
$$

For $N \in \mathbb{N}$, let

$$
M_{N}=\left\{k \in \mathbb{Z}_{+}^{n}: 1 \leq|k| \leq N\right\} .
$$

We shall use the following result, proven in [12].

Theorem 1 ([12], Theorem 2). Every symmetric continuous $N$-homogeneous polynomial $P:\left(L_{\infty}^{(\mathbb{C})}[0,1]\right)^{n} \rightarrow \mathbb{C}$ can be uniquely represented as

$$
P(y)=\sum_{\substack{l: M_{N} \rightarrow \mathbb{Z}_{+} \\ \varkappa\left(l, M_{N}\right)=N}} \alpha_{l} \prod_{\substack{k \in M_{N} \\ l(k)>0}}\left(R_{k}^{(\mathbb{C})}(y)\right)^{l(k)},
$$

where $y \in\left(L_{\infty}^{(\mathbb{C})}[0,1]\right)^{n}, \alpha_{l} \in \mathbb{C}, M_{N}$ is defined by (6), and $\varkappa$ is defined by (5). 
We shall use the following result, proven in [2].

Theorem 2 ([2], Theorem 3.1). For every sequence $\xi=\left\{\xi_{m}\right\}_{m=1}^{\infty} \subset \mathbb{C}$ such that

$$
\sup _{m \in \mathbb{N}} \sqrt[m]{\left|\xi_{m}\right|}<+\infty
$$

there exists $y_{\xi} \in L_{\infty}^{(\mathbb{C})}[0,1]$ such that

$$
\int_{[0,1]}\left(y_{\xi}(t)\right)^{m} d t=\xi_{m}
$$

for every $m \in \mathbb{N}$ and $\left\|y_{\xi}\right\|_{\infty} \leq \frac{2}{D} \sup _{m \in \mathbb{N}} \sqrt[m]{\left|\xi_{m}\right|}$, where

$$
D=\prod_{m=1}^{\infty} \cos \left(\frac{\pi}{2} \frac{1}{m+1}\right)
$$

2. The algebraic basis of the algebra of symmetric continuous real-valued polynomials on $\left(L_{\infty}^{(\mathbb{R})}[0,1]\right)^{n}$. For every $N$-homogeneous polynomial $P:\left(L_{\infty}^{(\mathbb{R})}[0,1]\right)^{n} \rightarrow \mathbb{R}$, let us define an $N$-homogeneous polynomial $\widehat{P}:\left(L_{\infty}^{(\mathbb{C})}[0,1]\right)^{n} \rightarrow \mathbb{C}$ in the following way. Let $A_{P}$ be the $N$-linear symmetric mapping associated with $P$. Let

$$
A_{\widehat{P}}: \underbrace{\left(L_{\infty}^{(\mathbb{C})}[0,1]\right)^{n} \times \ldots \times\left(L_{\infty}^{(\mathbb{C})}[0,1]\right)^{n}}_{N} \rightarrow \mathbb{C}
$$

be defined by

$$
A_{\widehat{P}}\left(y_{1}, \ldots, y_{N}\right)=\sum_{j_{1}=0}^{1} \ldots \sum_{j_{N}=0}^{1} i^{j_{1}+\ldots+j_{N}} A_{P}\left(y_{1}^{\left(j_{1}\right)}, \ldots, y_{N}^{\left(j_{N}\right)}\right)
$$

where $y^{(0)}$ and $y^{(1)}$ for every $y=\left(y_{1}, \ldots, y_{n}\right) \in\left(L_{\infty}^{(\mathbb{C})}[0,1]\right)^{n}$ are defined by $y^{(0)}(t)=$ $\left(\operatorname{Re} y_{1}(t), \ldots, \operatorname{Re} y_{n}(t)\right)$ and $y^{(1)}(t)=\left(\operatorname{Im} y_{1}(t), \ldots, \operatorname{Im} y_{n}(t)\right)$ for $t \in[0,1]$. It can be checked that $A_{\widehat{P}}$ is an $N$-linear symmetric mapping. Let

$$
\widehat{P}(y)=A_{\widehat{P}}(\underbrace{y, \ldots, y}_{N})
$$

for every $y \in\left(L_{\infty}^{(\mathbb{C})}[0,1]\right)^{n}$. Note that the polynomial $\widehat{P}$ is usually called the complexification of the polynomial $P$. It can be checked that for every $N_{1}$-homogeneous polynomial $P_{1}$ and for every $N_{2}$-homogeneous polynomial $P_{2}$, which act from $\left(L_{\infty}^{(\mathbb{R})}[0,1]\right)^{n}$ to $\mathbb{R}$, where $N_{1}, N_{2} \in \mathbb{N}$, we have $\widehat{P_{1} P_{2}}=\widehat{P_{1} P_{2}}$.

For every polynomial $P:\left(L_{\infty}^{(\mathbb{R})}[0,1]\right)^{n} \rightarrow \mathbb{R}$ of the form $(2)$, let

$$
\widehat{P}=P_{0}+\widehat{P}_{1}+\ldots+\widehat{P}_{N} .
$$

Proposition 1. For every symmetric continuous $N$-homogeneous polynomial $P$, acting from $\left(L_{\infty}^{(\mathbb{R})}[0,1]\right)^{n}$ to $\mathbb{R}$, the $N$-homogeneous polynomial $\widehat{P}:\left(L_{\infty}^{(\mathbb{C})}[0,1]\right)^{n} \rightarrow \mathbb{C}$, defined by $(8)$, is symmetric, continuous and

$$
\|\widehat{P}\| \leq \frac{(2 N)^{N}}{N !}\|P\|
$$


Proof. Let $P:\left(L_{\infty}^{(\mathbb{R})}[0,1]\right)^{n} \rightarrow \mathbb{R}$ be a symmetric continuous $N$-homogeneous polynomial. Let $A_{P}$ be the $N$-linear symmetric mapping associated with $P$.

By [1, p. 82, Formula 2],

$$
\|\widehat{P}\| \leq \frac{(2 N)^{N}}{N !}\|P\| .
$$

Therefore, the continuity of $P$ implies the continuity of $\widehat{P}$.

Since $P$ is symmetric, by Corollary 1 ,

$$
A_{P}\left(y_{1} \circ \sigma, \ldots, y_{N} \circ \sigma\right)=A_{P}\left(y_{1}, \ldots, y_{N}\right)
$$

for every $y_{1}, \ldots, y_{N} \in\left(L_{\infty}^{(\mathbb{R})}[0,1]\right)^{n}$ and $\sigma \in \Xi$. Therefore, by $(7)$,

$$
A_{\widehat{P}}\left(z_{1} \circ \sigma, \ldots, z_{N} \circ \sigma\right)=A_{\widehat{P}}\left(z_{1}, \ldots, z_{N}\right)
$$

for every $z_{1}, \ldots, z_{N} \in\left(L_{\infty}^{(\mathbb{C})}[0,1]\right)^{n}$ and $\sigma \in \Xi$. Consequently, by (8),

$$
\widehat{P}(z \circ \sigma)=A_{\widehat{P}}(\underbrace{z \circ \sigma, \ldots, z \circ \sigma}_{N})=A_{\widehat{P}}(\underbrace{z, \ldots, z}_{N})=\widehat{P}(z)
$$

for every $z \in\left(L_{\infty}^{(\mathbb{C})}[0,1]\right)^{n}$ and $\sigma \in \Xi$. Thus, $\widehat{P}$ is symmetric.

Proposition 2. The set of polynomials $\left\{R_{k}^{(\mathbb{R})}: k \in \mathbb{Z}_{+}^{n},|k| \geq 1\right\}$ is algebraically independent.

Proof. Let $N \in \mathbb{N}$. Let us show that the set $\left\{R_{k}^{(\mathbb{R})}: k \in M_{N}\right\}$ is algebraically independent, where $M_{N}$ is defined by (6). Suppose

$$
\alpha_{0}+\sum_{\mu=1}^{\mu^{\prime}} \sum_{\substack{l: M_{N} \rightarrow \mathbb{Z}_{+} \\ \varkappa\left(l, M_{N}\right)=\mu}} \alpha_{l} \prod_{\substack{k \in M_{N} \\ l(k)>0}}\left(R_{k}^{(\mathbb{R})}(y)\right)^{l(k)}=0
$$

for every $y \in\left(L_{\infty}^{(\mathbb{R})}[0,1]\right)^{n}$, where $\alpha_{0}, \alpha_{l} \in \mathbb{R}$ and $\mu^{\prime} \in \mathbb{N}$. Let us show that all the coefficients $\alpha_{0}, \alpha_{l}$ are equal to zero. For $\mu \in\left\{1, \ldots, \mu^{\prime}\right\}$, let

$$
Q_{\mu}(y)=\sum_{\substack{l: M_{N} \rightarrow \mathbb{Z}_{+} \\ \varkappa\left(l, M_{N}\right)=\mu}} \alpha_{l} \prod_{\substack{k \in M_{N} \\ l(k)>0}}\left(R_{k}^{(\mathbb{R})}(y)\right)^{l(k)}
$$

for $y \in\left(L_{\infty}^{(\mathbb{R})}[0,1]\right)^{n}$. Note that $Q_{\mu}$ is a $\mu$-homogeneous polynomial. By (10),

$$
\alpha_{0}+\sum_{\mu=1}^{\mu^{\prime}} Q_{\mu}(y)=0
$$

for every $y \in\left(L_{\infty}^{(\mathbb{R})}[0,1]\right)^{n}$. Therefore, by (3) and (11), $\alpha_{0}=0$ and $Q_{\mu}(y)=0$ for every $y \in\left(L_{\infty}^{(\mathbb{R})}[0,1]\right)^{n}$ and $\mu \in\left\{1, \ldots, \mu^{\prime}\right\}$. Consequently, $\left\|Q_{\mu}\right\|=0$ for every $\mu \in\left\{1, \ldots, \mu^{\prime}\right\}$. 
Therefore, by Proposition 1, $\left\|\widehat{Q}_{\mu}\right\|=0$ and, consequently, $\widehat{Q}_{\mu}$ is identically equal to zero. Taking into account $\widehat{R_{k}^{(\mathbb{R})}}=R_{k}^{(\mathbb{C})}$,

$$
\widehat{Q}_{\mu}(y)=\sum_{\substack{l: M_{N} \rightarrow \mathbb{Z}_{+} \\ \varkappa\left(l, M_{N}\right)=\mu}} \alpha_{l} \prod_{\substack{k \in M_{N} \\ l(k)>0}}\left(R_{k}^{(\mathbb{C})}(y)\right)^{l(k)}
$$

for $y \in\left(L_{\infty}^{(\mathbb{R})}[0,1]\right)^{n}$ and $\mu \in\left\{1, \ldots, \mu^{\prime}\right\}$. Since $\widehat{Q}_{\mu}$ is a symmetric continuous $\mu$-homogeneous polynomial on $\left(L_{\infty}^{(\mathbb{C})}[0,1]\right)^{n}$, by Theorem 1 , the representation $(12)$ is unique and, consequently, $\alpha_{l}=0$ for every $l: M_{N} \rightarrow \mathbb{Z}_{+}$such that $\varkappa\left(l, M_{N}\right)=\mu$. Thus, the set of polynomials $\left\{R_{k}^{(\mathbb{R})}: k \in M_{N}\right\}$ is algebraically independent.

Since every finite subset of the set $\left\{k \in \mathbb{Z}_{+}^{n}:|k| \geq 1\right\}$ is a subset of the set $M_{N}$ for some $N \in \mathbb{N}$, it follows that the set of polynomials $\left\{R_{k}^{(\mathbb{R})}: k \in \mathbb{Z}_{+}^{n},|k| \geq 1\right\}$ is algebraically independent.

Note that we cannot prove Proposition 2 by analogy to proof of [12, Proposition 1], because the proof of [12, Proposition 1] essentially uses [7, Theorem 6], which, in general, is not valid for the real case.

Let us prove the following analog of Theorem 1.

Theorem 3. Every symmetric continuous $N$-homogeneous polynomial $P:\left(L_{\infty}^{(\mathbb{R})}[0,1]\right)^{n} \rightarrow \mathbb{R}$ can be uniquely represented as

$$
P(y)=\sum_{\substack{l: M_{N} \rightarrow \mathbb{Z}_{+} \\ \varkappa\left(l, M_{N}\right)=N}} \alpha_{l} \prod_{\substack{k \in M_{N} \\ l(k)>0}}\left(R_{k}^{(\mathbb{R})}(y)\right)^{l(k)}
$$

where $y \in\left(L_{\infty}^{(\mathbb{R})}[0,1]\right)^{n}, \alpha_{l} \in \mathbb{R}, M_{N}$ is defined by (6), and $\varkappa$ is defined by (5).

Proof. Let $P:\left(L_{\infty}^{(\mathbb{R})}[0,1]\right)^{n} \rightarrow \mathbb{R}$ be a symmetric continuous $N$-homogeneous polynomial. Let $\widehat{P}:\left(L_{\infty}^{(\mathbb{C})}[0,1]\right)^{n} \rightarrow \mathbb{C}$ be the $N$-homogeneous polynomial, defined by (8). By Proposition 1, $\widehat{P}$ is symmetric and continuous. Therefore, by Theorem $1, \widehat{P}$ can be uniquely represented as

$$
\widehat{P}(y)=\sum_{\substack{l: M_{N} \rightarrow \mathbb{Z}_{+} \\ \varkappa\left(l, M_{N}\right)=N}} \alpha_{l} \prod_{\substack{k \in M_{N} \\ l(k)>0}}\left(R_{k}^{(\mathbb{C})}(y)\right)^{l(k)}
$$

where $y \in\left(L_{\infty}^{(\mathbb{C})}[0,1]\right)^{n}, \alpha_{l}$ are, in general, complex numbers, $M_{N}$ is defined by $(6)$, and $\varkappa$ is defined by $(5)$. For every $y \in\left(L_{\infty}^{(\mathbb{R})}[0,1]\right)^{n}$ we have $\widehat{P}(y)=P(y)$ and $R_{k}^{(\mathbb{C})}(y)=R_{k}^{(\mathbb{R})}(y)$ for $k \in M_{N}$. Therefore,

$$
P(y)=\sum_{\substack{l: M_{N} \rightarrow \mathbb{Z}_{+} \\ \varkappa\left(l, M_{N}\right)=N}} \alpha_{l} \prod_{\substack{k \in M_{N} \\ l(k)>0}}\left(R_{k}^{(\mathbb{R})}(y)\right)^{l(k)}
$$

for every $y \in\left(L_{\infty}^{(\mathbb{R})}[0,1]\right)^{n}$. Let us show that all the coefficients $\alpha_{l}$ are real numbers. Since $P(y) \in \mathbb{R}$, it follows that $P(y)-\overline{P(y)}=0$, i.e.,

$$
2 \sum_{\substack{l: M_{N} \rightarrow \mathbb{Z}_{+} \\ \varkappa\left(l, M_{N}\right)=N}} \operatorname{Im} \alpha_{l} \prod_{\substack{k \in M_{N} \\ l(k)>0}}\left(R_{k}^{(\mathbb{R})}(y)\right)^{l(k)}=0
$$


for every $y \in\left(L_{\infty}^{(\mathbb{R})}[0,1]\right)^{n}$. By Proposition 2, the set of polynomials $\left\{R_{k}^{(\mathbb{R})}: k \in M_{N}\right\}$ is algebraically independent. Therefore, by (14), $\operatorname{Im} \alpha_{l}=0$ for every coefficient $\alpha_{l}$. Moreover, since the set of polynomials $\left\{R_{k}^{(\mathbb{R})}: k \in M_{N}\right\}$ is algebraically independent, it follows that the representation (13) is unique.

Note that we cannot prove Theorem 3 by analogy to proof of Theorem 1, because the proof of Theorem 1 essentially uses Theorem 2, which, in general, is not valid for the real case.

Corollary 2. The set of polynomials

$$
\left\{R_{k}^{(\mathbb{R})}: k \in \mathbb{Z}_{+}^{n},|k| \geq 1\right\}
$$

is an algebraic basis of the algebra of all symmetric continuous real-valued polynomials on $\left(L_{\infty}^{(\mathbb{R})}[0,1]\right)^{n}$.

Proof. Let $P:\left(L_{\infty}^{(\mathbb{R})}[0,1]\right)^{n} \rightarrow \mathbb{R}$ be a symmetric continuous polynomial. Then $P$ can be represented in the form

$$
P=P_{0}+P_{1}+\ldots+P_{N^{\prime}}
$$

where $N^{\prime} \in \mathbb{N}, P_{0} \in \mathbb{R}$ and $P_{j}$ is a $j$-homogeneous polynomial for $j \in\left\{1, \ldots, N^{\prime}\right\}$. For every $j \in\left\{1, \ldots, N^{\prime}\right\}$, by $(3), P_{j}$ is symmetric and continuous. Therefore, by Theorem $3, P_{j}$ can be represented as an algebraic combination of elements of the set (15). Consequently, $P$ can be represented as an algebraic combination of elements of the set (15). By Proposition 2, the set (15) is algebraically independent. Consequently, the representation of $P$ as an algebraic combination of elements of the set (15) is unique.

3. Completion of the algebra of symmetric continuous real-valued polynomials on $L_{\infty}^{(\mathbb{R})}[0,1]$. Theorem 3 and Corollary 2 imply the following corollary.

Corollary 3. Every symmetric continuous polynomial $P: L_{\infty}^{(\mathbb{R})}[0,1] \rightarrow \mathbb{R}$ of degree at most $N^{\prime}$ can be uniquely represented as

$$
P(y)=\alpha_{0}+\sum_{N=1}^{N^{\prime}} \sum_{\substack{l_{1}, \ldots, l_{N} \in \mathbb{Z}_{+} \\ l_{1}+2 l_{2}+\ldots+N l_{N}=N}} \alpha_{l_{1}, \ldots, l_{N}} \prod_{\substack{k \in\{1, \ldots, N\} \\ l_{k}>0}}\left(R_{k}^{(\mathbb{R})}(y)\right)^{l_{k}}
$$

where $y \in L_{\infty}^{(\mathbb{R})}[0,1]$ and $\alpha_{l_{1}, \ldots, l_{N}} \in \mathbb{R}$. Consequently,

$$
\widehat{P}(y)=\alpha_{0}+\sum_{N=1}^{N^{\prime}} \sum_{\substack{l_{1}, \ldots, l_{N} \in \mathbb{Z}_{+} \\ l_{1}+2 l_{2}+\ldots+N l_{N}=N}} \alpha_{l_{1}, \ldots, l_{N}} \prod_{\substack{k \in\{1, \ldots, N\} \\ l_{k}>0}}\left(R_{k}^{(\mathbb{C})}(y)\right)^{l_{k}}
$$

where $y \in L_{\infty}^{(\mathbb{C})}[0,1]$

Let $G$ be a set of all functions $g \in L_{\infty}^{(\mathbb{C})}[0,1]$ such that $\bar{g}=g \circ \sigma_{g}$ for some $\sigma_{g} \in \Xi$, where $\bar{g}$ is the complex conjugate function to $g$.

Lemma 1. For every $m \in \mathbb{N}$ and $g \in G$, the value $R_{m}^{(\mathbb{C})}(g)$ is real. 
Proof. Let $m \in \mathbb{N}$ and $g \in G$. Then there exists $\sigma_{g} \in \Xi$ such that $\bar{g}=g \circ \sigma_{g}$. Since the polynomial $R_{m}^{(\mathbb{C})}$ is symmetric, it follows that

$$
R_{m}^{(\mathbb{C})}(\bar{g})=R_{m}^{(\mathbb{C})}\left(g \circ \sigma_{g}\right)=R_{m}^{(\mathbb{C})}(g) .
$$

On the other hand,

$$
R_{m}^{(\mathbb{C})}(\bar{g})=\overline{R_{m}^{(\mathbb{C})}(g)}
$$

Therefore, $\overline{R_{m}^{(\mathbb{C})}(g)}=R_{m}^{(\mathbb{C})}(g)$. Thus, $R_{m}^{(\mathbb{C})}(g)$ is real.

Let us denote $\mathcal{P}_{s}\left(L_{\infty}^{(\mathbb{R})}[0,1]\right)$ the algebra of all symmetric continuous polynomials, which act from $L_{\infty}^{(\mathbb{R})}[0,1]$ to $\mathbb{R}$.

Corollary 4. For every $P \in \mathcal{P}_{s}\left(L_{\infty}^{(\mathbb{R})}[0,1]\right)$ and $g \in G$, the value $\widehat{P}(g)$ is real.

Proof. This is an immediate consequence from Corollary 3 and Lemma 1.

For every $r \in \mathbb{N}$ and $P \in \mathcal{P}_{s}\left(L_{\infty}^{(\mathbb{R})}[0,1]\right)$, let

$$
\|P\|_{r}=\sup _{\substack{\|g\|_{\infty} \leq r \\ g \in G}}|\widehat{P}(g)| .
$$

Let $\mathcal{A}_{s}\left(L_{\infty}^{(\mathbb{R})}[0,1]\right)$ be the completion of $\mathcal{P}_{s}\left(L_{\infty}^{(\mathbb{R})}[0,1]\right)$ with respect to the metric

$$
d\left(P_{1}, P_{2}\right)=\sum_{r=1}^{\infty} \frac{1}{2^{r}} \frac{\left\|P_{1}-P_{2}\right\|_{r}}{1+\left\|P_{1}-P_{2}\right\|_{r}},
$$

where $P_{1}, P_{2} \in \mathcal{P}_{s}\left(L_{\infty}^{(\mathbb{R})}[0,1]\right)$, generated by a countable system of norms $\left\{\|\cdot\|_{r}: r \in \mathbb{N}\right\}$.

For every function $f \in \mathcal{A}_{s}\left(L_{\infty}^{(\mathbb{R})}[0,1]\right)$, let us define the function $\widehat{f}: G \rightarrow \mathbb{R}$ in the following way. Let $\left\{P_{m}\right\}_{m=1}^{\infty} \subset \mathcal{P}_{s}\left(L_{\infty}^{(\mathbb{R})}[0,1]\right)$ be a sequence of polynomials, which converges to $f$. For $g \in G$, let

$$
\widehat{f}(g)=\lim _{m \rightarrow \infty} \widehat{P}_{m}(g) .
$$

It can be checked that $\widehat{f}(g)$ does not depend on the choice of the sequence $\left\{P_{m}\right\}_{m=1}^{\infty}$. By Corollary $4, \widehat{P}_{m}(g) \in \mathbb{R}$, therefore, $\widehat{f}(g) \in \mathbb{R}$.

For every $r \in \mathbb{N}$ and $f \in \mathcal{A}_{s}\left(L_{\infty}^{(\mathbb{R})}[0,1]\right)$, let

$$
\|f\|_{r}=\sup _{\substack{\|g\|_{\infty} \leq r \\ g \in G}}|\widehat{f}(g)| .
$$

Note that $\mathcal{A}_{s}\left(L_{\infty}^{(\mathbb{R})}[0,1]\right)$ is a Fréchet algebra of functions, which act from $L_{\infty}^{(\mathbb{R})}[0,1]$ to $\mathbb{R}$, with respect to the metric

$$
d\left(f_{1}, f_{2}\right)=\sum_{r=1}^{\infty} \frac{1}{2^{r}} \frac{\left\|f_{1}-f_{2}\right\|_{r}}{1+\left\|f_{1}-f_{2}\right\|_{r}},
$$

where $f_{1}, f_{2} \in \mathcal{A}_{s}\left(L_{\infty}^{(\mathbb{R})}[0,1]\right)$. Note that a sequence of elements of $\mathcal{A}_{s}\left(L_{\infty}^{(\mathbb{R})}[0,1]\right)$ is convergent (fundamental) with respect to the metric (17) if and only if it is convergent (fundamental) with respect to every norm $\|\cdot\|_{r}, r \in \mathbb{N}$. 
Proposition 3. Let $f: L_{\infty}^{(\mathbb{C})}[0,1] \rightarrow \mathbb{C}$ be a symmetric analytic function, which is bounded on every bounded subset of $L_{\infty}^{(\mathbb{C})}[0,1]$, of the form

$$
f(y)=\alpha_{0}+\sum_{N=1}^{\infty} \sum_{\substack{l_{1}, \ldots, l_{N} \in \mathbb{Z}_{+} \\ l_{1}+2 l_{2}+\ldots+N l_{N}=N}} \alpha_{l_{1}, \ldots, l_{N}} \prod_{\substack{k \in\{1, \ldots, N\} \\ l_{k}>0}}\left(R_{k}^{(\mathbb{C})}(y)\right)^{l_{k}}
$$

where $y \in L_{\infty}^{(\mathbb{C})}[0,1]$ and all the coefficients $\alpha_{l_{1}, \ldots, l_{N}}$ are real. Then the restriction of the function $f$ to $L_{\infty}^{(\mathbb{R})}[0,1]$ belongs to the algebra $\mathcal{A}_{s}\left(L_{\infty}^{(\mathbb{R})}[0,1]\right)$.

Proof. For $m \in \mathbb{N}$, let $f_{m}: L_{\infty}^{(\mathbb{C})}[0,1] \rightarrow \mathbb{C}$ be defined by

$$
f_{m}(y)=\alpha_{0}+\sum_{N=1}^{m} \sum_{\substack{l_{1}, \ldots, l_{N} \in \mathbb{Z}_{+} \\ l_{1}+2 l_{2}+\ldots+N l_{N}=N}} \alpha_{l_{1}, \ldots, l_{N}} \prod_{\substack{k \in\{1, \ldots, N\} \\ l_{k}>0}}\left(R_{k}^{(\mathbb{C})}(y)\right)^{l_{k}}
$$

where $y \in L_{\infty}^{(\mathbb{C})}[0,1]$. Since $f$ is analytic and bounded on every bounded subset of $L_{\infty}^{(\mathbb{C})}[0,1]$, it follows that, for every $r \in \mathbb{N}$,

$$
\sup _{\substack{\|y\|_{\infty} \leq r \\ y \in L_{\infty}^{(\mathbb{C})}[0,1]}}\left|f(y)-f_{m}(y)\right| \rightarrow 0 \quad \text { as } \quad m \rightarrow \infty
$$

and

$$
\sup _{\substack{\|y\|_{\infty} \leq r \\ y \in L_{\infty}^{(\mathbb{C})}[0,1]}}\left|f_{m^{\prime}}(y)-f_{m^{\prime \prime}}(y)\right| \rightarrow 0 \quad \text { as } \quad \min \left\{m^{\prime}, m^{\prime \prime}\right\} \rightarrow \infty .
$$

For $m \in \mathbb{N}$, let $h_{m}$ be the restriction of $f_{m}$ to $L_{\infty}^{(\mathbb{R})}[0,1]$, i.e.,

$$
h_{m}(y)=\alpha_{0}+\sum_{N=1}^{m} \sum_{\substack{l_{1}, \ldots, l_{N} \in \mathbb{Z}_{+} \\ l_{1}+2 l_{2}+\ldots+N l_{N}=N}} \alpha_{l_{1}, \ldots, l_{N}} \prod_{\substack{k \in\{1, \ldots, N\} \\ l_{k}>0}}\left(R_{k}^{(\mathbb{R})}(y)\right)^{l_{k}},
$$

where $y \in L_{\infty}^{(\mathbb{R})}[0,1]$. Evidently, $\left\{h_{m}\right\}_{m=1}^{\infty} \subset \mathcal{P}_{s}\left(L_{\infty}^{(\mathbb{R})}[0,1]\right)$. For every $r \in \mathbb{N}$, by $(19)$,

$$
\begin{aligned}
\left\|h_{m^{\prime}}-h_{m^{\prime \prime}}\right\|_{r}= & \sup _{\substack{\|g\|_{\infty} \leq r \\
g \in G}}\left|\widehat{h}_{m^{\prime}}(g)-\widehat{h}_{m^{\prime \prime}}(g)\right|=\sup _{\substack{\|g\|_{\infty} \leq r \\
g \in G}}\left|f_{m^{\prime}}(g)-f_{m^{\prime \prime}}(g)\right| \\
& \leq \sup _{\substack{\|y\|_{\infty} \leq r \\
y \in L_{\infty}^{(\mathbb{C})}[0,1]}}\left|f_{m^{\prime}}(y)-f_{m^{\prime \prime}}(y)\right| \rightarrow 0
\end{aligned}
$$

as $\min \left\{m^{\prime}, m^{\prime \prime}\right\} \rightarrow \infty$. Consequently, the sequence $\left\{h_{m}\right\}_{m=1}^{\infty}$ is fundamental with respect to the metric (17). Since the algebra $\mathcal{A}_{s}\left(L_{\infty}^{(\mathbb{R})}[0,1]\right)$ is complete, it follows that there exists $h \in \mathcal{A}_{s}\left(L_{\infty}^{(\mathbb{R})}[0,1]\right)$ such that $\left\{h_{m}\right\}_{m=1}^{\infty}$ converges to $h$. Let us show that $h$ is the restriction of $f$ to $L_{\infty}^{(\mathbb{R})}[0,1]$. Let $y \in L_{\infty}^{(\mathbb{R})}[0,1]$. Let $r \in \mathbb{N}$ be such that $r \geq\|y\|_{\infty}$. Then $\left\|h-h_{m}\right\|_{r} \rightarrow 0$ as $m \rightarrow \infty$ and, consequently, the sequence $\left\{h_{m}(y)\right\}_{m=1}^{\infty}$ converges to $h(y)$. On the other hand, by (18), taking into account $f_{m}(y)=h_{m}(y)$, the sequence $\left\{h_{m}(y)\right\}_{m=1}^{\infty}$ converges to $f(y)$. Thus, $h(y)=f(y)$. 
The following proposition shows that the algebra $\mathcal{A}_{s}\left(L_{\infty}^{(\mathbb{R})}[0,1]\right)$ contains nonanalytic functions.

Proposition 4. The function

$$
L_{\infty}^{(\mathbb{R})}[0,1] \ni y \mapsto\left|R_{1}^{(\mathbb{R})}(y)\right| \in \mathbb{R}
$$

belongs to the algebra $\mathcal{A}_{s}\left(L_{\infty}^{(\mathbb{R})}[0,1]\right)$.

Proof. By the Stone-Weierstrass theorem, for every $r \in \mathbb{N}$, there exists the sequence of real-valued polynomials of one real variable $\left\{p_{m}^{(r)}\right\}_{m=1}^{\infty}$, uniformly convergent to the function

$$
\mathbb{R} \ni t \mapsto|t| \in \mathbb{R}
$$

on $[-r, r]$. Let $q_{m}=p_{m}^{(m)}$ for every $m \in \mathbb{N}$. Then the sequence $\left\{q_{m}\right\}_{m=1}^{\infty}$ is uniformly convergent to the function $(21)$ on every segment $[a, b] \subset \mathbb{R}$. For every $m \in \mathbb{N}$, let

$$
Q_{m}: L_{\infty}^{(\mathbb{R})}[0,1] \rightarrow \mathbb{R}
$$

be defined by

$$
Q_{m}(y)=q_{m}\left(R_{1}^{(\mathbb{R})}(y)\right),
$$

where $y \in L_{\infty}^{(\mathbb{R})}[0,1]$. Evidently, $Q_{m}$ is a symmetric continuous polynomial on $L_{\infty}^{(\mathbb{R})}[0,1]$ for every $m \in \mathbb{N}$. Let us show that the sequence $\left\{Q_{m}\right\}_{m=1}^{\infty}$ is fundamental in $\mathcal{A}_{s}\left(L_{\infty}^{(\mathbb{R})}[0,1]\right)$. For $r, m^{\prime}, m^{\prime \prime} \in \mathbb{N}$,

$$
\left\|Q_{m^{\prime}}-Q_{m^{\prime \prime}}\right\|_{r}=\sup _{\substack{\|g\|_{\infty} \leq r \\ g \in G}}\left|\widehat{Q}_{m^{\prime}}(g)-\widehat{Q}_{m^{\prime \prime}}(g)\right|=\sup _{\substack{\|g\|_{\infty} \leq r \\ g \in G}}\left|q_{m^{\prime}}\left(R_{1}^{(\mathbb{C})}(g)\right)-q_{m^{\prime \prime}}\left(R_{1}^{(\mathbb{C})}(g)\right)\right| .
$$

For every $g \in G$ such that $\|g\|_{\infty} \leq r$, we have $R_{1}^{(\mathbb{C})}(g) \in \mathbb{R}$ and

$$
\left|R_{1}^{(\mathbb{C})}(g)\right| \leq \int_{[0,1]}|g(t)| d t \leq\|g\|_{\infty} \leq r
$$

that is, $R_{1}^{(\mathbb{C})}(g) \in[-r, r]$. Consequently,

$$
\sup _{\substack{\|g\|_{\infty} \leq r \\ g \in G}}\left|q_{m^{\prime}}\left(R_{1}^{(\mathbb{C})}(g)\right)-q_{m^{\prime \prime}}\left(R_{1}^{(\mathbb{C})}(g)\right)\right| \leq \sup _{\substack{t \in[-r, r] \\ m^{\prime}}}\left|q_{m^{\prime}}(t)-q_{m^{\prime \prime}}(t)\right| .
$$

Since the sequence $\left\{q_{m}\right\}_{m=1}^{\infty}$ is uniformly convergent on $[-r, r]$, it follows that

$$
\sup _{t \in[-r, r]}\left|q_{m^{\prime}}(t)-q_{m^{\prime \prime}}(t)\right| \rightarrow 0 \quad \text { as } \quad \min \left\{m^{\prime}, m^{\prime \prime}\right\} \rightarrow \infty .
$$

By (23), (24) and (25), $\left\|Q_{m^{\prime}}-Q_{m^{\prime \prime}}\right\|_{r} \rightarrow 0$ as $\min \left\{m^{\prime}, m^{\prime \prime}\right\} \rightarrow \infty$. Thus, the sequence $\left\{Q_{m}\right\}_{m=1}^{\infty}$ is fundamental with respect to the norm $\|\cdot\|_{r}$ for every $r \in \mathbb{N}$. Consequently, $\left\{Q_{m}\right\}_{m=1}^{\infty}$ is fundamental with respect to the metric (17). Since the algebra $\mathcal{A}_{s}\left(L_{\infty}^{(\mathbb{R})}[0,1]\right)$ is complete, there exists $f \in \mathcal{A}_{s}\left(L_{\infty}^{(\mathbb{R})}[0,1]\right)$ such that $\left\{Q_{m}\right\}_{m=1}^{\infty}$ converges to $f$. Let us show that $f(y)=\left|R_{1}^{(\mathbb{R})}(y)\right|$ for every $y \in L_{\infty}^{(\mathbb{R})}[0,1]$. Let $y_{0} \in L_{\infty}^{(\mathbb{R})}[0,1]$. Let $r \in \mathbb{N}$ be such that $r \geq\left\|y_{0}\right\|_{\infty}$. Since the sequence $\left\{Q_{m}\right\}_{m=1}^{\infty}$ converges to $f$ with respect to the norm $\|\cdot\|_{r}$, 
it follows that the sequence $\left\{Q_{m}\left(y_{0}\right)\right\}_{m=1}^{\infty}$ converges to $f\left(y_{0}\right)$. By $(22), Q_{m}\left(y_{0}\right)=q_{m}\left(t_{0}\right)$, where $t_{0}=R_{1}^{(\mathbb{R})}\left(y_{0}\right)$. Then the sequence $\left\{q_{m}\left(t_{0}\right)\right\}_{m=1}^{\infty}$ converges to $f\left(y_{0}\right)$. Let $r_{0} \in \mathbb{N}$ be such that $t_{0} \in\left[-r_{0}, r_{0}\right]$. Since the sequence $\left\{q_{m}\right\}_{m=1}^{\infty}$ uniformly converges to the function (21) on $\left[-r_{0}, r_{0}\right]$, it follows that the sequence $\left\{q_{m}\left(t_{0}\right)\right\}_{m=1}^{\infty}$ converges to $\left|t_{0}\right|$. By the uniqueness of the limit, $f\left(y_{0}\right)=\left|t_{0}\right|$, i.e., $f\left(y_{0}\right)=\left|R_{1}^{(\mathbb{R})}\left(y_{0}\right)\right|$. Thus, $f(y)=\left|R_{1}^{(\mathbb{R})}(y)\right|$ for every $y \in L_{\infty}^{(\mathbb{R})}[0$, 1], i.e., the function (20) coincides with $f$. Consequently, the function (20) belongs to the algebra $\mathcal{A}_{s}\left(L_{\infty}^{(\mathbb{R})}[0,1]\right)$.

Let $\mathcal{M}$ be the set of all continuous multiplicative linear functionals $\varphi: \mathcal{A}_{s}\left(L_{\infty}^{(\mathbb{R})}[0,1]\right) \rightarrow \mathbb{R}$. In other words, $\mathcal{M}$ is the spectrum of the algebra $\mathcal{A}_{s}\left(L_{\infty}^{(\mathbb{R})}[0,1]\right)$. Let us describe the set $\mathcal{M}$.

Let $g \in G$. It can be checked that the mapping $\delta_{g}: \mathcal{A}_{s}\left(L_{\infty}^{(\mathbb{R})}[0,1]\right) \rightarrow \mathbb{R}$, defined by $\delta_{g}(f)=\widehat{f}(g)$, is a multiplicative linear functional. Also note that $\delta_{g}$ is continuous with respect to every norm $\|\cdot\|_{r}$, where $r \in \mathbb{N}$ and $r \geq\|g\|_{\infty}$. Thus, $\delta_{g} \in \mathcal{M}$.

Proposition 5. For every sequence $\xi=\left\{\xi_{m}\right\}_{m=1}^{\infty} \subset \mathbb{R}$ such that $\sup _{m \in \mathbb{N}} \sqrt[m]{\left|\xi_{m}\right|}<+\infty$, there exists $g_{\xi} \in G$ such that $R_{m}^{(\mathbb{C})}\left(g_{\xi}\right)=\xi_{m}$ for every $m \in \mathbb{N}$.

Proof. Let $\xi=\left\{\xi_{m}\right\}_{m=1}^{\infty} \subset \mathbb{R}$ be such that $\sup _{m \in \mathbb{N}} \sqrt[m]{\left|\xi_{m}\right|}<+\infty$. By Theorem 2, there exists $y_{\xi} \in L_{\infty}^{(\mathbb{C})}[0,1]$ such that $R_{m}^{(\mathbb{C})}\left(y_{\xi}\right)=\xi_{m}$ for every $m \in \mathbb{N}$. Let $g_{\xi}:[0,1] \rightarrow \mathbb{C}$ be defined by

$$
g_{\xi}(t)= \begin{cases}\overline{y_{\xi}(2 t)}, & \text { if } t \in\left[0, \frac{1}{2}\right) \\ y_{\xi}(2 t-1), & \text { if } t \in\left[\frac{1}{2}, 1\right] .\end{cases}
$$

Since $\bar{g}_{\xi}=g \circ \sigma$, where $\sigma:[0,1] \rightarrow[0,1]$ is defined by

$$
\sigma(t)= \begin{cases}t+\frac{1}{2}, & \text { if } t \in\left[0, \frac{1}{2}\right) \\ t-\frac{1}{2}, & \text { if } t \in\left[\frac{1}{2}, 1\right]\end{cases}
$$

it follows that $g_{\xi} \in G$. For every $m \in \mathbb{N}$,

$$
R_{m}^{(\mathbb{C})}\left(g_{\xi}\right)=\int_{0}^{\frac{1}{2}}\left(\overline{y_{\xi}(2 t)}\right)^{m} d t+\int_{\frac{1}{2}}^{1}\left(y_{\xi}(2 t-1)\right)^{m} d t=\frac{1}{2} \bar{\xi}_{m}+\frac{1}{2} \xi_{m}=\xi_{m}
$$

Proposition 6. For every $\varphi \in \mathcal{M}$

$$
\sup _{m \in \mathbb{N}} \sqrt[m]{\left|\varphi\left(R_{m}^{(\mathbb{R})}\right)\right|}<+\infty
$$

Proof. Since $\varphi$ is continuous, it follows that there exists $r \in \mathbb{N}$, such that $\varphi$ is continuous with respect to the norm $\|\cdot\|_{r}$. Therefore, there exists $C>0$ such that $|\varphi(f)| \leq C\|f\|_{r}$ for every $f \in \mathcal{A}_{s}\left(L_{\infty}^{(\mathbb{R})}[0,1]\right)$. Since

$$
\left\|R_{m}^{(\mathbb{R})}\right\|_{r}=\sup _{\substack{\|g\|_{\infty} \leq r \\ g \in G}}\left|\widehat{R}_{m}^{(\mathbb{R})}(g)\right|=\sup _{\substack{\|\|_{\infty} \leq r \\ g \in G}}\left|R_{m}^{(\mathbb{C})}(g)\right| \leq \sup _{\substack{\|g\|_{\infty} \leq r \\ g \in G}} \int_{0}^{1}|g(t)|^{m} d t \leq r^{m},
$$

it follows that

$$
\left|\varphi\left(R_{m}^{(\mathbb{R})}\right)\right| \leq C r^{m}
$$


for every $m \in \mathbb{N}$. Therefore,

$$
\sup _{m \in \mathbb{N}} \sqrt[m]{\left|\varphi\left(R_{m}^{(\mathbb{R})}\right)\right|}<+\infty
$$

Proposition 7. Let $\varphi, \psi \in \mathcal{M}$ be such that $\varphi\left(R_{m}^{(\mathbb{R})}\right)=\psi\left(R_{m}^{(\mathbb{R})}\right)$ for every $m \in \mathbb{N}$. Then $\varphi=\psi$.

Proof. By Corollary 3, every $P \in \mathcal{P}_{s}\left(L_{\infty}^{(\mathbb{R})}[0,1]\right)$ can be uniquely represented in the form (3). Therefore, since $\varphi$ and $\psi$ are multiplicative and linear,

$$
\begin{gathered}
\varphi(P)=\alpha_{0}+\sum_{N=1}^{N^{\prime}} \sum_{\substack{l_{1}, \ldots, l_{N} \in \mathbb{Z}_{+} \\
l_{1}+2 l_{2}+\ldots+N l_{N}=N}} \alpha_{l_{1}, \ldots, l_{N}} \prod_{\substack{k \in\{1, \ldots, N\} \\
l_{k}>0}}\left(\varphi\left(R_{k}^{(\mathbb{R})}\right)\right)^{l_{k}} \\
=\alpha_{0}+\sum_{N=1}^{N^{\prime}} \sum_{\substack{l_{1}, \ldots, l_{N} \in \mathbb{Z}_{+} \\
l_{1}+2 l_{2}+\ldots+N l_{N}=N}} \alpha_{l_{1}, \ldots, l_{N}} \prod_{\substack{k \in\{1, \ldots, N\} \\
l_{k}>0}}\left(\psi\left(R_{k}^{(\mathbb{R})}\right)\right)^{l_{k}}=\psi(P)
\end{gathered}
$$

for every $P \in \mathcal{P}_{s}\left(L_{\infty}^{(\mathbb{R})}[0,1]\right)$. Since $\varphi$ and $\psi$ are continuous and $\varphi$ coincides with $\psi$ on $\mathcal{P}_{s}\left(L_{\infty}^{(\mathbb{R})}[0,1]\right)$, which is dense in $\mathcal{A}_{s}\left(L_{\infty}^{(\mathbb{R})}[0,1]\right)$, it follows that $\varphi(f)=\psi(f)$ for every $f \in$ $\mathcal{A}_{s}\left(L_{\infty}^{(\mathbb{R})}[0,1]\right)$.

Let $\varphi \in \mathcal{M}$. By Proposition 6, the sequence $\left\{\varphi\left(R_{m}^{(\mathbb{R})}\right)\right\}_{m=1}^{\infty}$ is such that

$$
\sup _{m \in \mathbb{N}} \sqrt[m]{\left|\varphi\left(R_{m}^{(\mathbb{R})}\right)\right|}<+\infty
$$

Therefore, by Proposition 5, there exists $g \in G$ such that $R_{m}^{(\mathbb{C})}(g)=\varphi\left(R_{m}^{(\mathbb{R})}\right)$ for every $m \in \mathbb{N}$. Since $R_{m}^{(\mathbb{C})}(g)=\widehat{R}_{m}^{(\mathbb{R})}(g)=\delta_{g}\left(R_{m}^{(\mathbb{R})}\right)$, it follows that $\varphi\left(R_{m}^{(\mathbb{R})}\right)=\delta_{g}\left(R_{m}^{(\mathbb{R})}\right)$ for every $m \in \mathbb{N}$. Therefore, by Proposition $7, \varphi=\delta_{g}$. Thus, we have proved the following theorem.

Theorem 4. For every $\varphi \in \mathcal{M}$ there exists $g \in G$ such that $\varphi=\delta_{g}$. Moreover, for every $\varphi \in \mathcal{M}$,

$$
\sup _{m \in \mathbb{N}} \sqrt[m]{\left|\varphi\left(R_{m}^{(\mathbb{R})}\right)\right|}<+\infty
$$

and, conversely, for every sequence $\left\{\xi_{m}\right\}_{m=1}^{\infty} \subset \mathbb{R}$ such that $\sup _{m \in \mathbb{N}} \sqrt[m]{\left|\xi_{m}\right|}<+\infty$, there exists unique $\varphi \in \mathcal{M}$ such that $\varphi\left(R_{m}^{(\mathbb{R})}\right)=\xi_{m}$ for every $m \in \mathbb{N}$.

\section{REFERENCES}

1. M. Boiso, P. Hajek, Analytic approximations of uniformly continuous functions in real Banach spaces, Journal of Mathematical Analysis and Applications, 256 (2001), 80-98. doi:10.1006jmaa.2000.7291.

2. P. Galindo, T. Vasylyshyn, A. Zagorodnyuk, The algebra of symmetric analytic functions on $L_{\infty}$, Proceedings of the Royal Society of Edinburgh: Section A Mathematics, 147 (2017), №4, 743-761. doi:10.1017/S0308210516000287. 
3. P. Galindo, T. Vasylyshyn, A. Zagorodnyuk, Symmetric and finitely symmetric polynomials on the spaces $\ell_{\infty}$ and $L_{\infty}[0,+\infty)$, Mathematische Nachrichten, 291 (2018), №11-12, 1712-1726. doi:10.1002/mana.201700314.

4. P. Galindo, T. Vasylyshyn, A. Zagorodnyuk, Analytic structure on the spectrum of the algebra of symmetric analytic functions on $L_{\infty}$, RACSAM, 114 (2020), Article number 56. doi:10.1007/s13398020-00791-w.

5. M. González, R. Gonzalo, J.A. Jaramillo, Symmetric polynomials on rearrangement invariant function spaces, J. London Math. Soc., 59 (1999), №2, 681-697. doi:10.1112/S0024610799007164.

6. V. Kravtsiv, Algebraic basis of the algebra of block-symmetric polynomials on $\ell_{1} \oplus \ell_{\infty}$, Carpathian Math. Publ., 11 (2019), №1, 89-95. doi:10.15330/cmp.11.1.89-95.

7. V. Kravtsiv, T. Vasylyshyn, A. Zagorodnyuk, On algebraic basis of the algebra of symmetric polynomials on $\ell_{p}\left(\mathbb{C}^{n}\right)$, Journal of Function Spaces, 2017 (2017), Article ID 4947925, 8 p. doi:10.1155/2017/4947925.

8. J. Mujica, Complex Analysis in Banach Spaces, North Holland, 1986.

9. A.S. Nemirovskii, S.M. Semenov, On polynomial approximation of functions on Hilbert space, Mat. USSR Sbornik, 21 (1973), №2, 255-277. doi:10.1070/SM1973v021n02ABEH002016.

10. T. Vasylyshyn, Point-evaluation functionals on algebras of symmetric functions on $\left(L_{\infty}\right)^{2}$, Carpathian Math. Publ., 11 (2019), №2, 493-501. doi:10.15330/cmp.11.2.493-501.

11. T. Vasylyshyn, Symmetric polynomials on $\left(L_{p}\right)^{n}$, European Journal of Math., 6 (2020), №1, $164-178$. doi:10.1007/s40879-018-0268-3.

12. Vasylyshyn T.V. The algebra of symmetric polynomials on $\left(L_{\infty}\right)^{n}$, Mat. Stud., 52 (2019), №1, 71-85. doi:10.30970/ms.52.1.71-85

13. T. Vasylyshyn, A. Zagorodnyuk, Continuous symmetric 3-homogeneous polynomials on spaces of Lebesgue measurable essentially bounded functions, Methods of Functional Analysis and Topology, 24 (2018), №4, 381-398.

Vasyl Stefanyk Precarpathian National University, Ukraine taras.v.vasylyshyn@gmail.com

azagorodn@gmail.com 